\title{
Development of a universal internal positive control
}

\author{
Mark F Kavlick ${ }^{*, 1}$ \\ ${ }^{1}$ Counterterrorism \& Forensic Science Research Unit, Laboratory Division, Federal Bureau of Investigation, \\ 2501 Investigation Parkway, Quantico, VA 22135, USA
}

BioTechniques 65: 275-280 (November 2018) 10.2144/btn-2018-0034

Keywords: amplification inhibitors • exogenous control $\bullet$ internal positive control $\bullet$ IPC

Described here is a novel, universal exogenous internal positive control (IPC), which is fully synthetic for unparalleled quality control. The IPC was rationally designed, is small and efficiently amplified, has been successfully utilized alone or in triplex qPCR reactions, and is not crossreactive to human DNA or to any of the numerous nonhuman DNA samples tested. The IPC was sensitive to inhibition by at least four commonly encountered amplification inhibitors. It was used in a hydrolysis probe qPCR assay; however, it may be adaptable for other applications such as intercalating dye GPCR, PCR, isothermal amplification and reverse transcription-PCR.

An exogenous internal positive control (IPC) is often included in amplification reactions to confirm DNA amplification, detect false negatives, and qualitatively detect the presence of amplification inhibitory substances in a sample [1,2]. When properly designed, the IPC will always amplify in the absence of inhibitors in a fully functioning amplification reaction. By contrast, in the presence of certain inhibitors at sufficient concentration, IPC template amplification will be reduced or completely inhibited. However, an IPC is qualitative and cannot identify the inhibitor or its concentration. Importantly, the IPC should not interfere with amplification of the primary target sequence(s). Rather, it ensures quality control, indicating the need to reformulate amplification reaction components and/ or repurify or dilute a sample to ensure successful target amplification, by removing or reducing the inhibitor, respectively.

In the simplest form, for example, for PCR, intercalating dye qPCR and isothermal amplification, an IPC is a system that includes a DNA template and amplification primers. In probe-based qPCR assays the IPC will also contain a separate fluorescent probe that is specific and uniquely labeled for the IPC template, for example, the hydrolysis TaqMan probe. In alternative qPCR chemistries, the probe may be covalently linked to one of the amplification primers, for example, the Scorpions probe.

Since the IPC template is exogenous, it is not inherent to the sample be amplified. Rather, it is an additional component of the amplification reaction. The template may be competitive, that is, homologous to an amplification target of interest, but mutated to distinguish it from that target [3-8]. However, such IPC templates may directly compete with the target of interest for amplification primers, and therefore potentially affect amplification of the target, particularly those of low copy. Alternatively, the IPC template may be noncompetitive, that is, non homologous to the target but nonetheless a naturally occurring template, often chosen for convenience [9-11]. However, nonhomologous IPC templates must be carefully evaluated for use with each amplification target to ensure that its sequence is definitively not naturally found in the sample being tested as well as to reduce the risk of spurious amplification products, which increases with ever larger IPC templates.

An ideal IPC template might be nonhomologous to any natural amplification target (universal), short in length for optimal amplification efficiency and to reduce the risk of spurious products, and easily prepared or commercially available for convenience. Examples of commercially available stand- alone IPCs exist [10,12-14]; although cost and/or assay incompatibility, for example, the fluorophore utilized, may exclude their use. As a case in point, a commercial exogenous IPC was successfully utilized in a duplex qPCR assay for quantifying human mtDNA [15,16]; however, subsequent development toward a triplex assay [17] prohibited its use due to dye emission overlap. In addition, custom modifications of a commercial IPC product, for example, an alternative reporter dye and/or alternative quencher, may be available at additional cost or not an option. Given the relative dearth of commercial IPC products, development of the universal IPC described here was undertaken.

\section{Materials \& methods}

\section{IPC design}

IPC template design included several criteria:

- Short length, e.g., $\leq 100$ bp, for optimal amplification efficiency [18];

- 50\% GC content of amplicon and primers to ensure amplification efficiency and to prevent primer mismatch stabilization [19];

- Random sequence having minimal homology to known natural sequences;

- Amenability to hydrolysis probes.

\section{METHOD SUMMARY}

A novel, universal exogenous internal positive control (IPC) may detect amplification inhibitors and false-negative results in qPCR reactions. It is fully synthetic, efficiently amplified, multiplex compatible, and offers greater flexibility compared with commercially available IPCs. 
Random 100-bp DNA sequences having $0.5 \mathrm{GC}$ content were generated using an online tool [20]. Generated sequences were evaluated as qPCR targets with Primer Express v3.0 software (Applied Biosystems, CA, USA) using the default settings. Where possible, sequences were manually trimmed at either end to evaluate ever shorter template sequences and were further trimmed to remove sequences distal to primer binding sites. Such sequences were confirmed again with Primer Express, then subjected to Blastn searches (NCBI) [21] to detect potential homology to natural sequences. After several candidate templates were evaluated in this manner, one 65-bp template sequence was identified that exhibited little homology to NCBI database entries and was highly compact, containing only six bps extraneous to the primer and probe binding sites (Figure 1).

\section{qPCR}

IPC forward and reverse primers were HPLC purified (Integrated DNA Technologies, IA, USA) and reconstituted with Tris EDTA buffer (TE) as 100- $\mu \mathrm{M}$ stocks. The IPC probe was NED labeled, contained a $3^{\prime}$ minor groove binder nonfluorescent quencher (MGB-NFQ), and was supplied at $100 \mu \mathrm{M}$ in TE (Applied Biosystems, CA, USA). IPC primer and probe assay concentrations initially tested in singleplex assays were 900 and $250 \mathrm{nM}$, respectively. Subsequently, 50-nM IPC forward primer, 300-nM IPC reverse primer and 250-nM IPC probe, were determined optimal for TaqMan assays, following the manufacturer's (Applied Biosystems) instructions, and utilized for triplex assays involving crossreactivity and inhibitor testing. Triplex qPCR assays also included primers and probes for two separate amplification targets, which were prepared and optimized similarly as described elsewhere [17].
The double-stranded (ds) IPC template consisted of two complementary, PAGEpurified synthetic oligonucleotides (Ultramers; Integrated DNA Technologies, IA, USA), which were combined in equimolar proportions. Briefly, the oligonucleotides were reconstituted to $100 \mu \mathrm{M}$ in TE and their concentrations were verified by absorbance at $260 \mathrm{~nm}$ using the extinction coefficients 784,600 and $768,500 \mathrm{l} /($ mole $\cdot \mathrm{cm})$, for the forward and reverse strands, respectively. The oligonucleotides were mixed in equal proportions to yield the ds IPC template, which was diluted in TE to 1250 copies/ $\mu$ l for regular IPC reactions or in a standard series described below for determining IPC amplification efficiency.

IPC QPCR was performed with $2 \mu$ l of sample DNA or TE as a no template control (NTC) in a $20 \mu$ l reaction containing primers, probe, IPC template and TaqMan 2X Fast Advanced Master Mix (Applied Biosystems). Primers and probe for other amplification targets were included in triplex QPCR assays. Reaction set up is provided in Table 1 and the full protocol for the universal IPC is available online as supplementary material at Biotechniques. com.

GPCR reactions were amplified on a 7500 Real Time PCR System (Applied Biosystems) as a custom assay using HID Real Time PCR Software V1.2 and the following standard conditions: $2 \mathrm{~min}$ at $50^{\circ} \mathrm{C}, 20 \mathrm{~s}$ at $95^{\circ} \mathrm{C}$ followed by 40 cycles of $3 \mathrm{~s}$ at $95^{\circ} \mathrm{C}$ and $30 \mathrm{~s}$ at $60^{\circ} \mathrm{C}$. Data were analyzed using a $0.2 \Delta R n$ cycle threshold (Ct) value and the automatic baseline algorithm. Amplification efficiency was calculated using the slope of the standard plot regression line: efficiency $\left.=\left[10^{(-1 / \mathrm{m})}\right]\right]^{-1}$.

Analysis of qPCR inhibition was qualitative wherein uninhibited samples were defined as those that had IPC Ct values comparable to those of the NTCs within the assay. Partially inhibited samples were defined as those having detectably higher Nostoc sp. PCC 7107

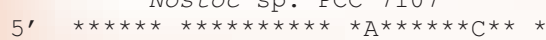

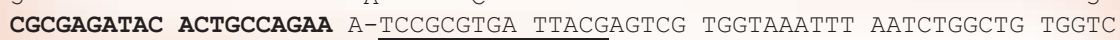
GCGCTCTATG TGACGGTCTT T-AGGCGCACT AATGCTCAGC ACCATTTAAA TTAGACCGAC ACCAG $3^{\prime}$ 20 30

Figure 1. Schematic of the universal internal positive control. Shown are the positions of the forward and reverse primers (bold) and probe (underlined) relative to the double-stranded internal positive control (IPC) template (49.2\% GC content). Also shown are coincidental, although inconsequential, partial homologies of the template to a Nostoc and a Citrus sequence. The Nostoc sequence was imperfectly homologous to 27 bases of the template and included an ' $A$ ' insertion following the 21st position and a ' $C$ ' mismatch at the 28th position. The Citrus sequence was perfectly homologous to 21 bases of the template. A chimeric template sequence similar to this IPC template is not expected to naturally exist and thus poses no risk for endogenous amplification in any sample.
Ct values and completely inhibited samples were those that exhibited undetermined IPC Ct values, that is, fluorescent signal was not detected.

\section{Cross-reactivity testing}

33 different commercially available species DNA preparations, including those from humans and other primates, other mammals, fish, birds, fungi and bacteria, were tested as samples, each at $100 \mathrm{ng}$ per qPCR reaction. Preparations included: chimpanzee (NIGMS Human Genetic Cell Repository at the Coriell Institute for Medical Research, NJ, USA: \#NS06006); baboon, bovine, cat, chicken, cynomolgus monkey, deer, dog, donkey, equine, ferret, fish, gerbil, goat, guinea pig, hamster, human, monkey, mouse, porcine, rabbit, rat, Rhesus monkey, sheep and turkey (Zyagen Laboratories, CA, USA); and Aspergillus oryzae, Bacillus subtilis, Candida albicans, Clostridium perfringens, Escherichia coli, Pseudomonas aeruginosa, Staphylococcus epidermidis, and Streptococcus pneumonia (American Type Culture Collection [ATCC], VA, USA).

\section{Inhibitor testing}

The IPC within the context of a triplex mtDNA QPCR assay [17] was evaluated against a panel of 11 commonly encountered PCR inhibitors. Inhibitor stock solutions were prepared in molecular biology-grade water, unless otherwise indicated, as follows: bile salts (Sigma-Aldrich, MO, USA \#B8756) at $1.2 \mathrm{mg} / \mathrm{ml}$, collagen (Sigma-Aldrich) at $8 \mathrm{mg} / \mathrm{ml}$ of $0.1 \mathrm{~N}$ acetic acid, $0.5 \mathrm{M}$ EDTA disodium salt solution (Sigma-Aldrich, \#E7889), guanidine $\mathrm{HCl}$ (Sigma-Aldrich \#G4505) at $12 \mathrm{mg} / \mathrm{ml}$, guanidine thiocyanate (Sigma-Aldrich, \#G9277) at $12 \mathrm{mg} / \mathrm{ml}$, hematin (Sigma-Aldrich, porcine) at $4.2 \mathrm{mM}$ prepared in $0.1 \mathrm{~N}$ sodium hydroxide, humic acid (Alfa Aesar, MA, USA) at $4 \mathrm{mg} / \mathrm{ml}$, indigo (Acros Organics, NJ, USA) at $10 \mathrm{mg} /$ $\mathrm{ml}$, melanin (MP Biomedicals, OH, USA) at $4 \mathrm{mg} / \mathrm{ml} 0.5 \mathrm{~N}$ sodium hydroxide, phenol (Fisher Scientific, \#A92) at $42 \mathrm{mM}$, and tannic acid (Alfa Aesar) at 1 and $10 \mathrm{mg} / \mathrm{ml}$. Stock solutions were diluted further in water and $2 \mu \mathrm{l}$ of various dilutions were subjected to triplex qPCR [17]. Amplification was considered inhibited when IPC Ct values shifted higher compared with assay controls. Partial inhibition was observed when Ct values increased beyond background and complete inhibition when $\mathrm{Ct}$ values were undetermined. 
Table 1. Optimized universal IPC qPCR reaction.

\begin{tabular}{|l|l|}
\hline Volume & \multicolumn{2}{l|}{ Reaction component } \\
\hline $0.8 \mu \mathrm{l}$ & IPC forward primer (50 $\mathrm{nM}$ final concentration) \\
\hline $0.8 \mu \mathrm{l}$ & IPC reverse primer (300 $\mathrm{nM}$ final concentration) \\
\hline $0.8 \mu \mathrm{l}$ & IPC probe (250 $\mathrm{nM}$ final) \\
\hline $0.8 \mu \mathrm{l}$ & IPC ds template (1250 copies/ $\mu$ l; 1000 copies/reaction) \\
\hline $10 \mu \mathrm{l}$ & TaqMan 2X fast advanced master mix (Applied Biosystems) \\
\hline $2.4 \mu \mathrm{l}$ & Primers and probe for amp target \#1, i.e., duplex qPCR ${ }^{\dagger}$ \\
\hline $2.4 \mu \mathrm{l}$ & Primers and probe for amp target \#2, i.e., triplex qPCR \\
\hline Q.S. & Molecular biology-grade water \\
\hline $18 \mu \mathrm{l}$ & NA \\
\hline $2 \mu \mathrm{l}$ & Sample, no template control, or dilution of a DNA standard \\
\hline $20 \mu \mathrm{l}$ & Total reaction volume \\
\hline $\begin{array}{l}{ }^{\dagger} \text { Optional duplex and triplex qPCR reaction components. } \\
\text { ds: Double-stranded; IPC: Internal positive control. }\end{array}$ & \\
\hline
\end{tabular}

\section{Results \& discussion}

The amplification efficiency of the IPC was assessed to ensure reliable amplification of the IPC template and its utility as a positive amplification control. Toward this end, the ds IPC template was serially diluted and amplified as a DNA standard in qPCR reactions using the IPC primers and probe at concentrations following the manufacturer's guidelines for the Fast Advanced Master Mix, that is, 900 and $250 \mathrm{nM}$, respectively. The resulting standard curve exhibited an $R^{2}$ value of 0.999, indicating a very low fraction of unexplained variance, and a slope of -3.448 , which corresponded to a high amplification efficiency of $94.999 \%$ (Figure 2). The $Y$ intercept of this plot (38.742) indicated that the IPC could theoretically amplify 1 copy/ $\mu$ l of IPC ds template within 40 amplification cycles, suggesting a high degree of sensitivity.

Subsequent to determination of high IPC amplification efficiency, IPC primer and probe concentrations were optimized for minimum $\mathrm{Ct}$ and maximum $\Delta \mathrm{Rn}$ following the manufacturer's instructions [22], as were primers and probe for each of two unrelated mtDNA amplification targets [17]. All three primer and probe sets were incorporated in a triplex qPCR reaction mixture at their optimal concentrations and IPC amplification efficiency was once again assessed using dilutions of the ds IPC template as the standard. The resultant IPC standard curve revealed that the IPC amplification efficiency was virtually unchanged in the optimized triplex reaction, relative to the singleplex IPC assay, at 94.365\% (Figure 3). Similar to the singleplex IPC standard curve, the triplex plot also exhibited a high $R^{2}$ value of 0.999 and $Y$ intercept below
40 amplification cycles indicating no loss of IPC sensitivity in the triplex assay. Based on these results, a mid-range IPC template copy number, that is, 1000 copies/reaction, was chosen to yield a relatively low IPC Ct value and ensure the utility of the IPC as a positive control.

The IPC was further evaluated within the context of the triplex assay described above, but using HL60 DNA (ATCC) for the standard curve in order to evaluate the amplification efficiencies of the two mtDNA targets. Five separate experiments revealed that efficiencies were similar to IPC amplification efficiency; mtDNA target 1 was $91.7-93.4 \%$ efficient and target 2 was 94.8-96.3\% efficient (data not shown). The results demonstrate their compatibility with the IPC in the triplex assay. The optimized IPC qPCR assay reaction is provided in Table 1 and a detailed protocol is available in the supplementary material.

The IPC, within the context of the described triplex assay, was then examined to detect possible crossreactivity to DNA preparations from 33 different extant species including human, chimpanzee and other primates, other mammals, fish, birds, fungi and bacteria. If any of the preparations naturally contained a DNA sequence that was similar to the IPC template, secondary IPC primer and probe binding and amplification could occur, to generate spurious products manifested by detectable increases in fluorescence above that of IPC template amplification. That is, crossreactivity would be detected by a shift toward lower IPC Ct values in the species DNA samples relative to those observed with NTCs. The results revealed that IPC Ct values were unchanged (data not shown) thus demonstrating no crossreactivity to the species tested here, in full concordance with the Blastn search described earlier. Given the experimental results and prior database searches, the IPC described here is empirically and theoretically universal, and might be successfully used as a truly nonhomologous positive control for any amplification target for any species sample. However, prior to fully employing the IPC for use with any prospective amplification target, end users are cautioned to test the IPC for crossreactivity to the sample types being amplified as well as for potential crossreactivity to the primers and probe of the amplification target(s).

Finally, the IPC, within the context of the described triplex assay, was tested against 11 commonly encountered PCR inhibitors. The results showed that the IPC detected complete inhibition at $40 \mu \mathrm{g} / \mathrm{ml}$ humic acid, $50 \mu \mathrm{g} / \mathrm{ml}$ tannic acid, $20 \mu \mathrm{g} / \mathrm{ml}$ melanin and 10 mM EDTA; although partial inhibition could be detected at lower concentrations as indicated by increased Ct values relative to the lowest concentrations (Figure 4). Inhibition was not observed for the maximal final reaction inhibitor concentrations of $80 \mu \mathrm{g} / \mathrm{ml}$ collagen, $42 \mu \mathrm{M}$ hematin, $100 \mu \mathrm{g} /$ $\mathrm{ml}$ indigo, $12 \mu \mathrm{g} / \mathrm{ml}$ bile salts, $120 \mu \mathrm{g} / \mathrm{ml}$ guanidinium thiocyanate, $120 \mu \mathrm{g} / \mathrm{ml}$ guanidinium $\mathrm{HCl}$ and $420 \mu \mathrm{M}$ phenol (data not shown). The results for the latter group of inhibitors were consistent for the Fast Advanced Master Mix used here, which has been found to overcome certain inhibitors [Betts G, Applied Biosystems, Pers. Comm.] as have other kits [23]. Therefore, the IPC may yet exhibit susceptibility to some or all of the remaining seven inhibitors when used with qPCR master mixes or components that are not specifically 


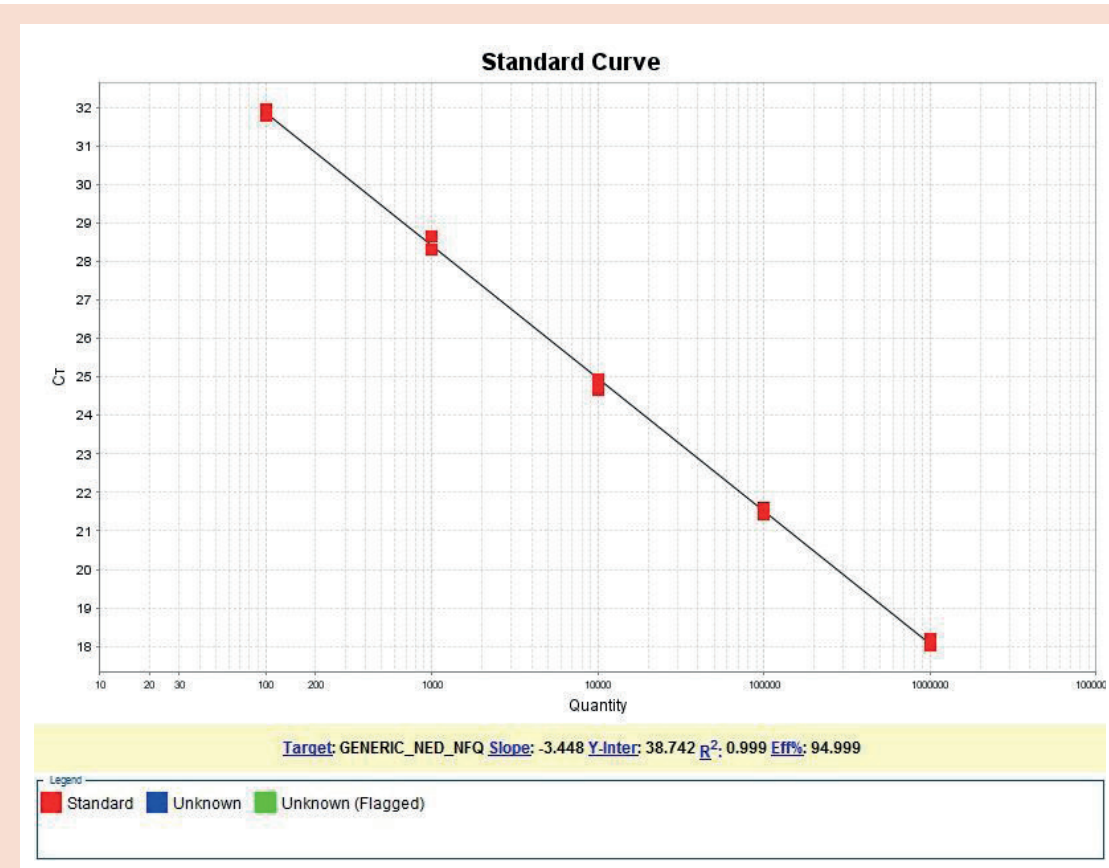

Figure 2. Internal positive control amplification efficiency in singleplex assay. To determine amplification efficiency of the internal positive control (IPC) reaction, assay components were prepared similar to that shown in Table 1, except for the primer $(900 \mathrm{mM})$ and probe $(250 \mathrm{nM})$ concentrations. In addition, primers and probes for amplification targets 1 and 2 were not included nor was the IPC template shown at 1000 copies/reaction. Instead, IPC template was serially diluted tenfold from $10^{2}-10^{6} \mathrm{copies} / \mu \mathrm{l}$ and used to create the standard curve shown (each dilution at $2 \mu \mathrm{l} /$ reaction, in duplicate). designed to overcome amplification inhibition. Further testing is warranted.
It is noteworthy that the short $65 \mathrm{bp}$ length of the IPC template did not appear to factor into the aforementioned inhibition

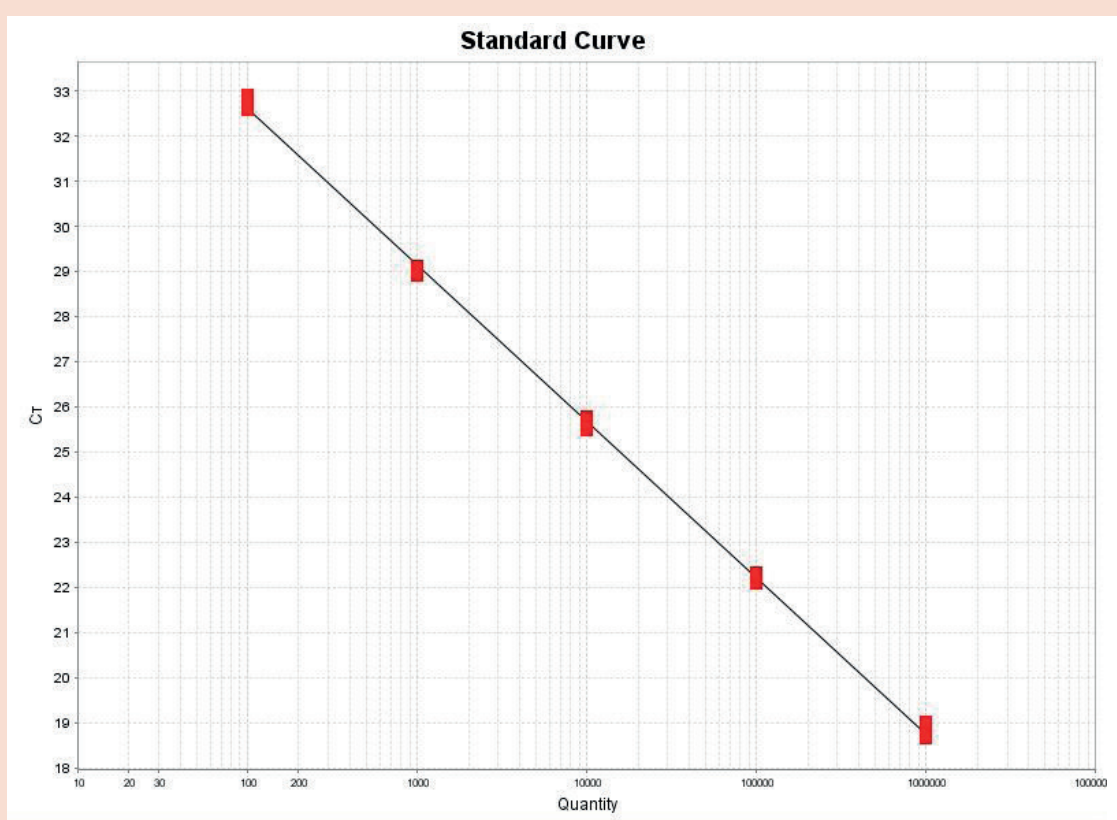

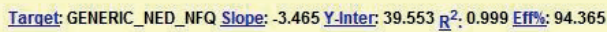

Figure 3. Internal positive control amplification efficiency in triplex assay. To determine internal positive control (IPC) amplification efficiency within a triplex reaction, assay components were prepared as shown in Table 1 and detailed elsewhere [17]. As with the singleplex assay (Figure 2), the standard quantity of double-stranded IPC template was replaced with tenfold serial dilutions from $10^{2}-10^{6}$ copies/ $\mu$ to create a standard curve. Two mtDNA amplification targets were concomitantly amplified (data not shown) from a fixed amount of $\mathrm{HL} 60$ included in the assay. profile, consistent with some [23], but not other [24] reports, since the FAM and VIC probes, which detected amplification of 105- and 316-bp mtDNA templates, respectively, yielded virtually comparable results for this panel of 11 inhibitors, that is, susceptibility to the same four inhibitors (Figure 4) but not the remaining seven (data not shown). Furthermore, template GC content, also suggested to affect inhibition [24], did not appear to factor in these results since all three targets were similar in GC content (45.3-49.2\%). Moreover, it is also notable that similar results for humic acid, tannic acid, melanin and EDTA were observed following PCR amplification of human mtDNA control region sequences (Daniels D, Laboratory Division, FBI, Pers. Comm.). Taken together, these observations suggest that the IPC can efficiently detect amplification inhibition within a sample and therefore the need to re-purify or dilute a sample to eliminate that inhibition.

While the IPC was successfully utilized in a triplex hydrolysis probe assay, similar performance in a quadruplex or higher multiplexed assay might be expected. The IPC might also be adapted for intercalating dye qPCR, such as SYBR green, isothermal amplification and PCR by eliminating the IPC probe. In the latter, the recommended IPC ds template concentration (Table 1) is expected to yield detectable PCR product ( $>6 \mathrm{ng} / \mu \mathrm{l}$ ) following $\geq 32$ amplification cycles based on its mass and assuming a 95\% amplification efficiency (calculation not shown). Additional development of the IPC for such applications is warranted, particularly with respect to optimization of IPC primer concentrations.

Yet another potential application are RT-PCR assays that utilize exogenous RNA IPCs. Like qPCR, the control RNA could be homologous to the target but mutated [25-27] or nonhomologous but natural [27-31], each with risk as described above. Therefore future developmental efforts might also include adapting the described IPC for use as a universal, nonhomologous exogenous internal control for RT-PCR. One suggested approach might involve RNA synthesis of the forward and/or reverse IPC template (Figure 1) with base modifications, such as phosphorothioate bonds [32], to convey resistance to degradation by pervasive RNases and ensure positivity of the control, similar in concept to IPC 

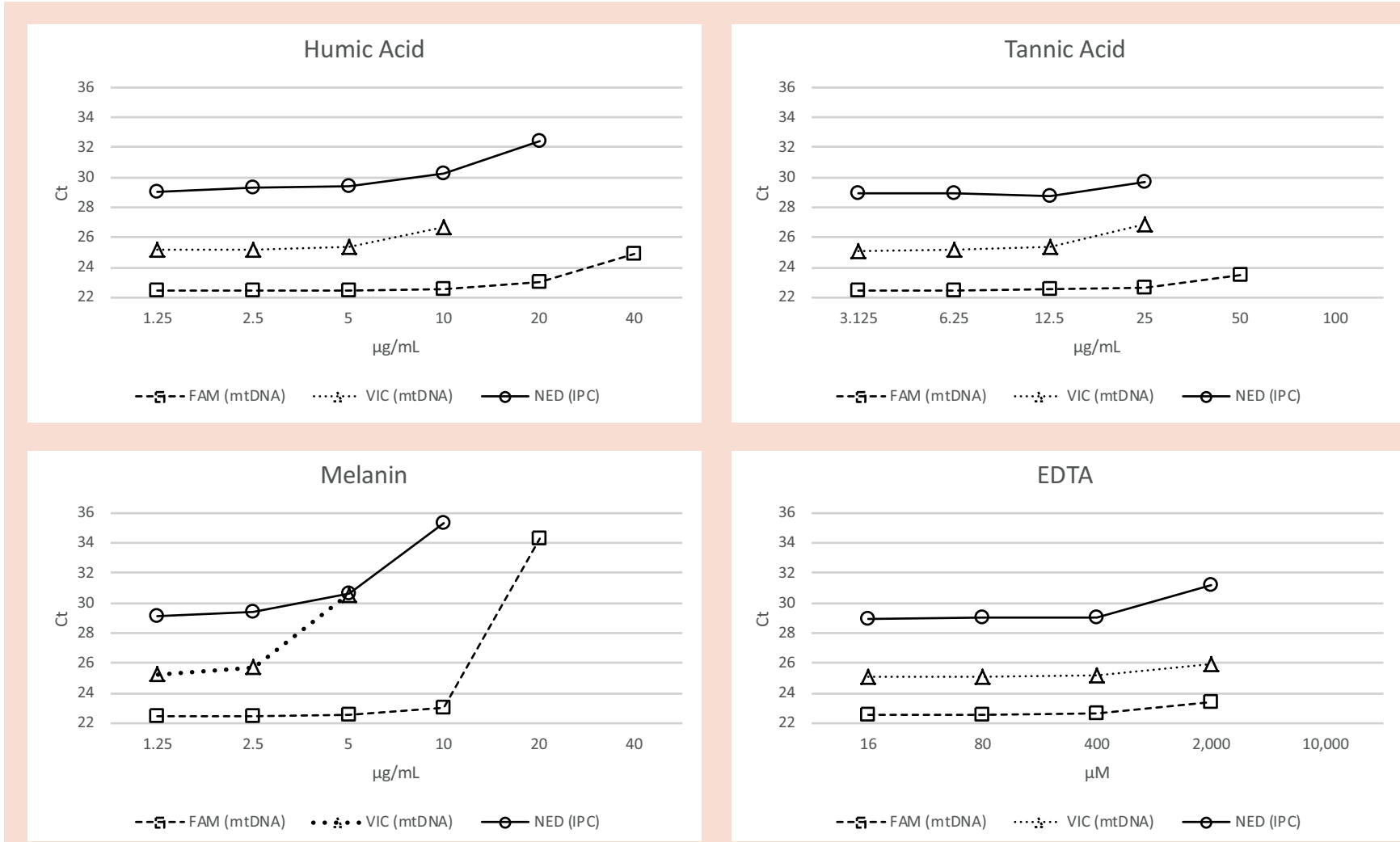

Figure 4. Internal positive control sensitivity to inhibitors. The internal positive control (IPC; NED probe signal) within the context of a triplex qPCR assay [17] was evaluated against a panel of 11 commonly encountered PCR inhibitors. The IPC was sensitive to humic acid, tannic acid, melanin and EDTA. Two mtDNA amplification targets - FAM signal for a 105-bp target and VIC signal for a 316-bp target - exhibited similar sensitivities. Ct values not present, i.e., undetermined, indicate full sensitivity of the signal to the higher inhibitor concentrations shown. The remaining seven inhibitors did not inhibit any of the probes at the highest concentrations tested (see text; data not shown). HL60 cell line DNA (ATCC) was added to each reaction to provide a template for the two mtDNA amplification targets to produce the FAM and VIC signals.

RNA templates constructed with a degradation protective protein shell [26,27,31]. The IPC RNA template would be spiked into a sample with subsequent RNA extraction and purification followed by a one- or two-step RT-PCR involving cDNA synthesis via random primers or the IPC primer(s) (Figure 1) and qPCR (Table 1). The universal exogenous control could also be employed in addition to an endogenous RT-PCR control in relative quantification, auch as a housekeeping gene, as a means to verify effective extraction of sample RNA.

The universal IPC offers the convenience and quality of a fully synthetic DNA template $[15,33,34]$ as well as a potential cost saving relative to commercial IPCs. Another advantage is flexibility of design, for instance, intercalating dye or hydrolysis probe qPCR, including choice of reporter dye and quencher in the latter. Yet another is the constancy of IPC probe signal in the triplex assay, which fell within the narrow range of 29-32 Ct for uninhibited samples within 60+ separate experiments (data not shown). It is also noteworthy that IPC signal was not inhibited by high levels of competing amplification targets, that is, 'inhibition by competition', which has been previously observed [15]; however, this feature may be attributable to the multiplex optimized nature of the Fast Advanced Master Mix [35] utilized and not to the IPC itself. These features combined with the amplification efficiency, universality, and inhibitor sensitivity of the IPC, support its use in qPCR.

\section{Acknowledgments}

The author would like to acknowledge Danielle L Daniels and Lilliana I Moreno for helpful discussions. This is publication number 18-17 of the Federal Bureau of Investigation (FBI) Laboratory. Names of commercial manufacturers are provided for identification purposes only and inclusion does not imply endorsement by the FBI. The views expressed are those of the author and do not necessarily reflect the official policy or position of the FBI or the US Government.

\section{Supplementary data}

To view the supplementary data that accompany this paper please visit the journal website at: www.future-science. com/doi/full/10.2144/btn-2018-0034

\section{Competing \& financial interests disclosure}

The author has no relevant affiliations or financial involvement with any organization or entity with a financial interest in or financial conflict with the subject matter or materials discussed in the manuscript. This includes employment, consultancies, honoraria, stock ownership or options, expert testimony, grants or patents received or pending, or royalties.

No writing assistance was utilized in the production of this manuscript.

\section{Open access}

This work is licensed under the CCO 1.0 Universal Public Domain Dedication 
License. To view a copy of this license, visit http://creativecommons.org/publicdomain/ zero/1.0/

\section{References}

1. Hoorfar J, Malorny B, Abdulmawjood A, Cook N, Wagner M, Fach P. Practical considerations in design of internal amplification controls for diagnostic PCR assays. J. Clin. Microbiol. 42(5), 1863-1868 (2004)

2. Phillips JM. Chapter 2. Real-time RT-PCR: What lies beneath the surface. In: A-Z of Quantitative $P C R$. Bustin SA (Ed.). International University Line, La Jolla, CA, USA, 47-85 (2004).

3. Herwegh S, Carnoy C, Wallet F, Loïez C, Courcol RJ. Development and use of an internal positive control for detection of Bordetella pertussis by PCR. J. Clin. Microbiol. 43(5), 2462-2464 (2005).

4. Jothikumar P, Hill V, Narayanan J. Design of FRET-TaqMan probes for multiplex real-time PCR using an internal positive control. Biotechniques 46(7), 519-524 (2009).

5. Ni W, Le Guiner C, Moullier P, Snyder RO. Development and utility of an internal threshold control (ITC) real-time PCR assay for exogenous DNA detection. PLoS One 7(5), e36461 (2012).

6. Majidzadeh K, Mohseni A, Soleimani M. Construction and evaluation of a novel internal positive control (IPC) for detection of Coxiella burnetii by PCR. Jundishapur J. Microbiol. 7(1), e8849 (2014).

7. Vinayagamoorthy T, Maryanski D, Vinayagamoorthy $D$ et al. Improved internal control for molecular diagnosis assays. MethodsX 2, 159164 (2015).

8. Hartleif S, Göhring K, Goelz R, Jahn G, Hamprecht K. Quantitative monitoring of HCMV DNAlactia in human milk by real time PCR assay: Implementation of internal control contributes to standardization and quality control. J. Virol. Methods 237, 101-106 (2016).

9. Hartman LJ, Coyne SR, Norwood DA. Development of a novel internal positive control for Taqman based assays. Mol. Cell. Probes 19(1), 51-59 (2005)

10. Burrel S, Fovet C, Brunet $\mathrm{C}$ et al. Routine use of duplex real-time PCR assays including a commercial internal control for molecular diagnosis of opportunistic DNA virus infections. J. Virol. Methods 185(1), 136-141 (2012).

11. Crannell ZA, Rohrman B, Richards-Kortum R. Development of a quantitative recombinase polymerase amplification assay with an internal positive control. J. Vis. Exp. 97, e52620 (2015).

12. Focus Diagnostics. www.focusdx.com/pdfs/pi/ US/MOL9000.pdf
13. Eurogentec. www.anaspec.com/content/ pdfs/c_literature183.pdf

14. $\operatorname{TaqMan}^{\circledR}$ Exogenous Internal Positive Control Reagents. www3.appliedbiosystems.com/cms/ groups/mcb_support/documents/generaldocuments/cms_041040.pdf

15. Kavlick MF, Lawrence HS, Merritt RT et al. Quantification of human mitochondrial DNA using synthesized DNA standards. J. Forensic Sci. 56(6), 1457-1463 (2011).

16. Sprouse ML, Phillips NR, Kavlick MF, Roby RK. Internal validation of human mitochondrial DNA quantification using real-time PCR. J. Forensic Sci. 59(4), 1049-1056 (2014).

17. Kavlick MF. Development of a triplex mtDNA qPCR assay to assess quantification, degradation, inhibition, and amplification target copy numbers. Mitochondrion (Accepted manuscript available online: 25 September 2018 doi:10.1016/j.mito.2018.09.007)

18. Debode F, Marien A, Janssen E, Bragard C, Berben $G$. The influence of amplicon length on real-time PCR results. Biotechnol. Agron. Soc. Environ. 21(1), 3-11 (2017).

19. Real-time PCR handbook. http://find.lifetechnologies.com/Global/FileLib/qPCR/RealTimePCR Handbook_Update_FLR.pdf

20. University of California Riverside. faculty.ucr. edu/ mmaduro/random.htm

21. Johnson M, Zaretskaya I, Raytselis Y, Merezhuk Y, McGinnis S, Madden TL. NCBI BLAST: a better web interface. Nucleic Acids Res. 36(Web Server issue), W5-W9 (2008).

22. TaqMan ${ }^{\circledR}$ Fast Advanced Master Mix Protocol. www3.appliedbiosystems.com/cms/groups/ mcb_support/documents/generaldocuments/ cms_084554.pdf

23. Mulero JJ, Chang CW, Lagacé RE et al. Devel opment and validation of the AmpFISTR MiniFiler PCR Amplification Kit: a MiniSTR multiplex for the analysis of degraded and/or PCR inhibited DNA. J. Forensic Sci. 53(4), 838-852 (2008).

24. Pionzio AM, McCord BR. The effect of internal control sequence and length on the response to PCR inhibition in real-time PCR quantitation. Forensic Sci. Int. Genet. 9, 55-60 (2014).

25. Chen Q, Hu Z, Zhang Q, Yu M. Development and evaluation of a real-time method of simultaneous amplification and testing of enterovirus 71 incorporating a RNA internal control system. J. Virol. Methods 196, 139-144 (2014).

26. Felder E, Wölfel R. Development of a versatile and stable internal control system for RT-qPCR assays. J. Virol. Methods 208, 33-40 (2014).

27. Zambenedetti MR, Pavoni DP, Dallabona AC et al. Internal control for real-time polymerase chain reaction based on MS2 bacteriophage for RNA viruses diagnostics. Mem. Inst. Oswaldo Cruz 112(5), 339-347 (2017).

28. Monpoeho S, Coste-Burel M, Costa-Mattiol $\mathrm{M}$ et al. Application of a real-time polymerase chain reaction with internal positive control for detection and quantification of enterovirus in cerebrospinal fluid. Eur. J. Clin. Microbiol. Infect. Dis. 21(7), 532-536 (2002).

29. Di Trani L, Bedini B, Donatelli I et al. A sensitive one-step real-time PCR for detection of avian influenza viruses using a MGB probe and an internal positive control. BMC Infect. Dis. 6, 87 (2006).

30. Sidoti F, Bergallo M, Terlizzi ME et al. Development of a quantitative real-time nucleic acid sequence-based amplification assay with an internal control using molecular beacon probes for selective and sensitive detection of human rhinovirus serotypes. Mol. Biotechnol. 50(3), 221-228 (2012).

31. Zhao L, Li R, Liu A, Zhao S. A novel duplex real time quantitative reverse transcription polymerase chain reaction for rubella virus with armored RNA as a noncompetitive interna positive control. J. Virol. Methods 219, 84-89 (2015).

32. Phosphorothioate Bonds Modifications. www.idtdna.com/site/Catalog/Modifications/ Category/8

33. Honeycutt R, Sobral BW, McClelland M Polymerase chain reaction (PCR) detection and quantification using a short PCR product and a synthetic internal positive control. Anal. Biochem. 248(2), 303-306 (1997).

34. Conte J, Potoczniak MJ, Tobe SS. Using synthetic oligonucleotides as standards in probe-based qPCR. Biotechniques 64(4), $177-$ 179 (2018)

35. TaqMan ${ }^{\circledR}$ Fast Advanced Master Mix. www thermofisher.com/content/dam/LifeTech/ Documents/PDFs/PG1397-PJ6767 C0010149-TaqMan-FastAdvancedMasterMixProductBulletin-Americas-FLR.pdf

First draft submitted: 4 April 2018; Accepted for publication: 9 August 2018

Address correspondence to: Mark F Kavlick Counterterrorism \& Forensic Science Research Unit, Laboratory Division, Federal Bureau of Investigation, 2501 Investigation Parkway, Quantico, VA 22135, USA; Mfkavlick@fbi.gov

To purchase reprints of this article contact: s.cavana@future-science.com

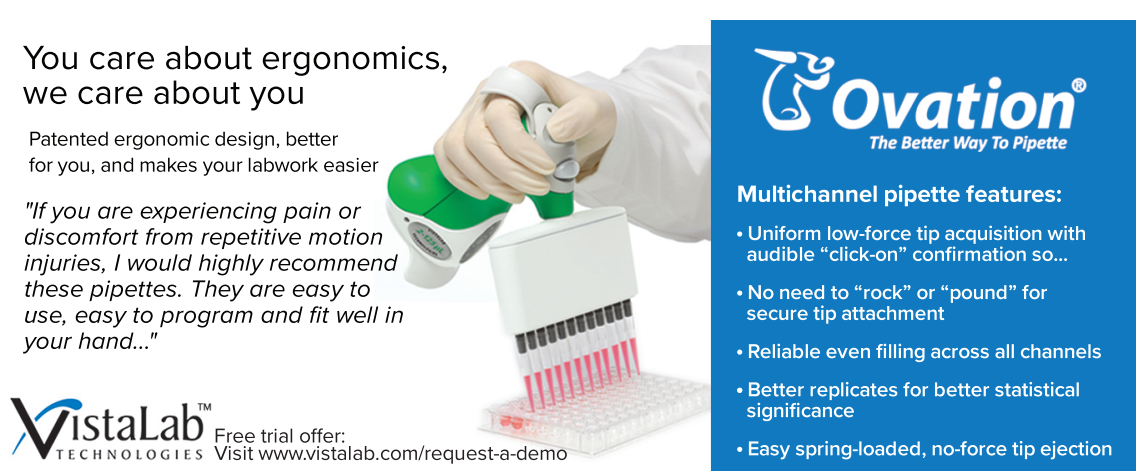


